\title{
In depth study of molybdenum silicon compound formation at buried interfaces
}

Erwin Zoethout, Eric Louis, and Fred Bijkerk

Citation: Journal of Applied Physics 120, 115303 (2016); doi: 10.1063/1.4962541

View online: https://doi.org/10.1063/1.4962541

View Table of Contents: http://aip.scitation.org/toc/jap/120/11

Published by the American Institute of Physics

\section{Articles you may be interested in}

Spectral tailoring of nanoscale EUV and soft x-ray multilayer optics

Applied Physics Reviews 4, 011104 (2017); 10.1063/1.4978290

In vacuo growth studies of $\mathrm{Ru}$ thin films on $\mathrm{Si}, \mathrm{SiN}$, and $\mathrm{SiO}_{2}$ by high-sensitivity low energy ion scattering Journal of Applied Physics 120, 065303 (2016); 10.1063/1.4960577

Ion acceleration in the transparent regime and the critical influence of the plasma density scale length Physics of Plasmas 23, 093117 (2016); 10.1063/1.4962571

A model for pressurized hydrogen induced thin film blisters Journal of Applied Physics 120, 235304 (2016); 10.1063/1.4972221

Review Article: Recommended reading list of early publications on atomic layer deposition-Outcome of the "Virtual Project on the History of ALD"

Journal of Vacuum Science \& Technology A: Vacuum, Surfaces, and Films 35, 010801 (2017); $10.1116 / 1.4971389$

$\mathrm{Cr} / \mathrm{B}_{4} \mathrm{C}$ multilayer mirrors: Study of interfaces and X-ray reflectance Journal of Applied Physics 119, 125307 (2016); 10.1063/1.4944723

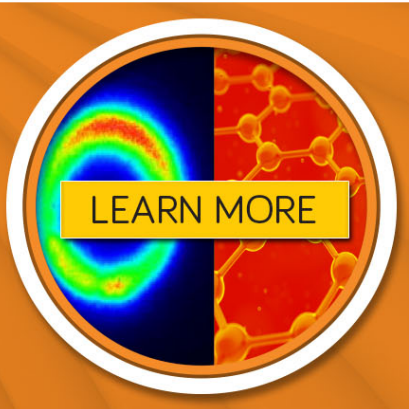




\title{
In depth study of molybdenum silicon compound formation at buried interfaces
}

\author{
Erwin Zoethout, ${ }^{1}$ Eric Louis, ${ }^{2}$ and Fred Bijkerk ${ }^{2}$ \\ ${ }^{1}$ FOM-DIFFER, P.O. Box 6336, 5600 HH Eindhoven, The Netherlands \\ ${ }^{2}$ Industrial Focus Group XUV Optics, MESA+ Institute for Nanotechnology, University of Twente, \\ P.O. Box 217, 7500 AE Enschede, The Netherlands
}

(Received 7 July 2016; accepted 29 August 2016; published online 16 September 2016)

\begin{abstract}
Angle resolved x-ray photoelectron spectroscopy (ARXPS) has been employed to determine nondestructively the in-depth interface formation during thin film growth. Buried interfaces underneath the nanometer thick layers are probed by identifying the chemical shift of compound materials in photoelectron spectroscopy and using the angular response to quantify the compound amounts from the measured intensities. The thin interfaces in molybdenum-silicon multilayers grown at ambient temperature are investigated. This system is an example of an almost perfect 1D-system, where the interface region is only a small part of the individual layer thicknesses of 3 to $5 \mathrm{~nm}$. Despite the low growth temperature, both the interfaces of this multilayer show layer thickness dependent interface formation. While the silicon-on-molybdenum interface shows a limited interface thickness of $0.4 \mathrm{~nm}$ of $\mathrm{Mo}_{5} \mathrm{Si}_{3}$, the molybdenum-on-silicon interface shows a more complex evolution. For this interface, the composition of the first $2.0 \mathrm{~nm}$ of deposited layer thickness is best described as a molybdenum-silicon compound layer with a molybdenum rich top and a $\mathrm{MoSi}_{2}$ bottom layer. After $2.5 \mathrm{~nm}$ of the deposited layer thickness, the molybdenum rich compound at the top has transformed into polycrystalline molybdenum on top of $1.8 \mathrm{~nm} \mathrm{MoSi} i_{2}$ at the interface. The formation of the $1.8 \mathrm{~nm} \mathrm{MoSi} 2$ precedes the formation of polycrystalline molybdenum on top. Angle resolved x-ray photoelectron spectroscopy (ARXPS) is shown to be a good tool to study the interface phenomena beneath the nanometer thick top layers. In the case of Mo/Si multilayer mirrors, this ARXPS study shows that the compound formation at the interface accounts for the majority of the extreme ultraviolet reflectance loss. Published by AIP Publishing.
\end{abstract}

[http://dx.doi.org/10.1063/1.4962541]

\section{INTRODUCTION}

The study of the composition of an interface during formation below a top layer that is only a few nanometers thick is inherently difficult because it is often outside the range of surface sensitive techniques (scanning tunneling microscopy (STM), atomic force microscopy, low energy ion scattering, auger electron spectroscopy, and low energy electron microscopy) and a marginal fraction of more bulk like techniques (Rutherford backscattering, energy dispersive x-ray spectroscopy, and x-ray diffraction (XRD)). In order to investigate these interfaces in systems where the only inhomogeneity in composition is in-depth, angle resolved $\mathrm{x}$-ray photoemission spectroscopy can be a powerful tool. The probing depth of this technique is determined by the attenuation length of the escaping electrons used for spectroscopy and, for the used monochromatic $\mathrm{Al}-\mathrm{K}_{\alpha}$ radiation, is of the order of $1.5-3 \mathrm{~nm}$, depending on the composition of the system under investigation. This medium surface sensitivity enables to detect both the top and substrate layers over a range of top layer thickness of several times the attenuation length. The possibility to identify chemical states and quantify the amounts of top layer material makes $\mathrm{x}$-ray photoemission spectroscopy a suitable tool to study the buried interfaces below a few nanometer thin layers.

A good example of such a 1D-system of thin layers is a molybdenum-silicon multilayer. Mo/Si multilayers are the enabling elements of the optical systems applied in extreme ultraviolet photolithography (EUVL), employing radiation with a wavelength of $13.5 \mathrm{~nm}$. In order to achieve a high reflectance, positive interference of the reflections from all the interfaces is required (thus reflecting a specific wavelength), and alternating layers of molybdenum and silicon are used with individual layer thicknesses of 3 to $5 \mathrm{~nm}$. Although a reflection of $75 \%$ is theoretically possible in this artificial Bragg reflector, imperfect interfaces between the two materials are mainly responsible for a limited practical reflectivity. Layer growth at room temperature has been shown to achieve optimal performance for $\mathrm{Mo} / \mathrm{Si}$ multilayers. ${ }^{1-3}$ A procedure for periodic noble gas ion treatment of the silicon layers after the deposition has resulted in a reflectivity of $69 \%$, ${ }^{4,5}$ which is close to the theoretical maximum. In-situ x-ray reflectometry, used to monitor layer deposition, has enabled an impression of the interface roughness development during the build-up of multilayer systems. Interlayer and crystallite formation in molybdenum silicon multilayers have been studied ex-situ extensively using grazing-incidence X-ray reflectivity (XRR), $\mathrm{X}$-ray diffraction (XRD), and transmission electron microscopy (TEM). ${ }^{1-11}$ From these studies, it has been concluded that the polycrystalline nature of the molybdenum layers is the main cause of interlayer roughness. Furthermore, these studies conclude that the interface region between the molybdenum and silicon layers of this room temperature grown multilayer occupies a volume up to $20 \%$ of the multilayer 
period. Recently, we reported on a study of direct measurement of the surface morphology during the deposition process. ${ }^{12}$ We have shown that the periodic ion treatment of the silicon layers in the multilayer production procedure is capable of reducing growth induced height differences down to the morphology of the first ion treated silicon layer, reducing the need of an interface study of this multilayer to the interface study of bi-layers. Furthermore, for the growth of molybdenum-on-silicon, the height differences evolving with increasing amounts are lacking behind compared to the expected values for polycrystalline growth. This most likely is due to compound formation at the interfaces. The interfaces have been investigated on crystalline substrates extensively with the help of dedicated surface science equipment. Near ambient temperature, an approximately $0.5 \mathrm{~nm}$ thick $\mathrm{MoSi}_{2}$ interface layer is formed. Only after a deposited amount of $2 \mathrm{~nm}$ layer, closure is suspected. ${ }^{13}$ At growth temperatures between 400 and $700^{\circ} \mathrm{C}$ and sub-monolayer amounts of molybdenum deposit, $\mathrm{MoSi}_{2}$ island growth has been reported on crystalline silicon substrates, with islands elevating $1.2 \mathrm{~nm}$ above the surface ${ }^{14}$ Both are exemplary for the complexities that can arise during interface formation in molybdenum silicon systems.

In this work, we investigate the deposition of both the multilayer components in vacuo with angle resolved x-ray photoelectron spectroscopy (ARXPS) to provide insight into the nanometer scale processes of the buried interface below the nanometers thick layers. By quantifying both the layer morphology and composition at different stages of the deposition process for dimensions down to the nanometer scale, the "intermixed" zone between two materials can be estimated. All the deposited layers are prepared under relevant conditions by using a deposition set-up that is also used for the development of multilayer films for EUV optics. The in vacuo approach prevents the exposure to the atmosphere, which usually modifies the top layer to a significant part of the XPS probing depth, both in composition as well as in morphology. The chemical shift in XPS data for molybdenum silicon systems will be explored by investigating room temperature grown mixed-molybdenum-silicon $\left(\mathrm{Mo}_{\mathrm{x}} \mathrm{Si}\right)$ films, with $\mathrm{x}$ in the range of $0.1-4$. The resulting identification of molybdenum-silicon compound formation will be used in the discussion on the silicon-on-molybdenum and molybdenumon-silicon interface formation. Investigation of the top morphology and modelling of the ARXPS data of bi-layer systems will show that even at growth temperatures as low as room temperature, compound formation between molybdenum and silicon is a dynamic process determined by the deposited amount.

\section{EXPERIMENTAL DETAILS}

Throughout the paper, molybdenum and silicon bi-layers as well as mixed-molybdenum-silicon films are deposited at room temperature onto the native oxide of super-polished silicon substrates in an ultra-high vacuum (UHV) environment better than $10^{-8}$ mbar. Silicon and molybdenum were deposited by electron beam evaporation. A Kaufman type ${ }^{15}$ hot cathode ion source, providing $100 \mathrm{eV}$ krypton ions, was used to modify the surface of freshly deposited silicon layers when silicon was used as a substrate layer. A fluence of $1.5 \times 10^{16}$ ions $/ \mathrm{cm}^{2}$ under a $45^{\circ}$ angle of incidence was used, resulting in the removal of $0.5 \mathrm{~nm}$ of silicon. Quartz crystal oscillator microbalances were used to control the amount of the deposited material, with an accuracy better than $1 \%$ of the reported value. Amounts (and rates) are reported as layer thickness assuming bulk density. Constant deposition rates are employed for both the materials during bi-layer formation at a rate of $0.025 \mathrm{~nm} / \mathrm{s}$. The samples were transported from the coating facility via a vacuum transfer system, base pressure $1 \times 10^{-9}$ mbar, to be analyzed with the aid of either a scanning tunneling microscope (STM, Leiden Probe Microscopy) or x-ray photoelectron spectroscopy (XPS, Thermo Fisher Scientific Theta Probe). The composition of the top surface was studied in a vacuum environment of $1 \times 10^{-9}$ mbar. Monochromatic $\mathrm{Al}-\mathrm{K}_{\alpha}$ radiation has been used to investigate the surface. For quantification, XPS sensitivity factors from the Scofield library are used ${ }^{16}$ together with attenuation lengths ${ }^{17}$ that are determined using bulk densities. Finally, machine specific calibration factors are applied (e.g., analyzer transmission function and source-detector geometry). The Theta Probe XPS used in this study employs an angle resolved lens, enabling measurements from different analyzer angles simultaneously from the same footprint. This lens type is routinely used to analyze a smooth, thin film in, for example, high- $\mathrm{k} / \mathrm{semiconductor}$ interfaces. ${ }^{18,19}$ It enables a relative fast measurement from a wide angular range. Photoelectrons measured by more grazing angles originate from a shallower depth, making them more surface sensitive compared to more normal angles. Comparing top layer/substrate layer intensity ratios at different angles allows in-depth concentration modelling of the different components. This only holds true for flat films. The sensitivity of the angular response to the morphology is determined by the angular binning of the detector. Throughout this paper, the $60^{\circ}$ acceptance angle of the analyzer is divided into 8 sub-regions. In this paper, the same division of analyzer angles is used as reported previously on the influence of surface morphology on ARXPS measurements of nanometer thin overlayers. ${ }^{20}$ The most grazing angle regions (all angles above $60^{\circ}$ ) are discarded ${ }^{21}$ because of the potentially large contribution of elastic scattering or the lack of intensity of one of the components. ARXPS data were analyzed with the Avantage software (Thermo Fisher Scientific), but the three-layer-model or box-model ${ }^{22}$ used in this paper is described in the supplementary material. The best model fit to the data always matches the measured data within the statistical noise of the measurement. The supplementary material also describes the relation to the reported error bars in more detail. For this paper, the acquisition time was chosen such that statistical noise allowed a relative intensity error to be in the range of $1 \%-10 \%$. The STM chamber was operated at a base pressure of $1 \times 10^{-9}$ mbar as well. The STM imaging was performed with mechanically cut Pt/Ir tips, using a sample bias of $2 \mathrm{~V}$ and a current of $0.2 \mathrm{nA}$ as typical tunnel parameters. STM performance was verified on highly ordered pyrolytic graphite (HOPG), where the observed atomic spacing was used for lateral calibration, and on polycrystalline gold, where the step heights on the grains were used to calibrate the heights. In order to prevent the possible influences 

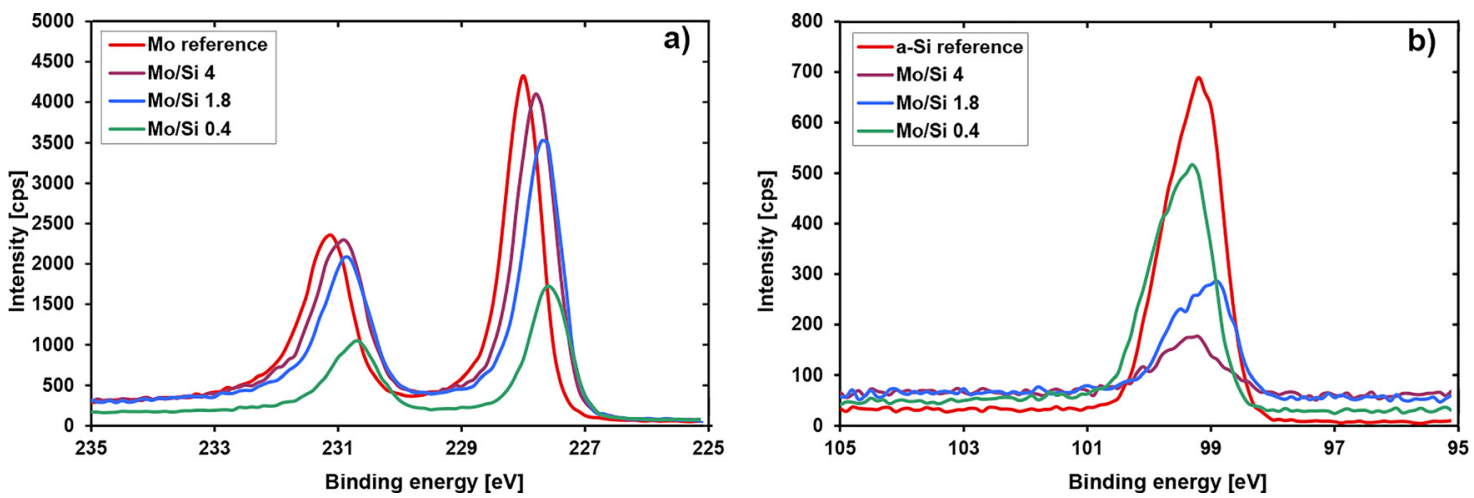

FIG. 1. Mo3d (a) and Si2p (b) peaks of the co-deposited molybdenum silicide layers $\left(\mathrm{Mo}_{\mathrm{x}} \mathrm{Si}\right.$ ) and of the reference layers of polycrystalline molybdenum and amorphous silicon.

on the film growth by previously deposited layers and/or prolonged exposure to residual gas during transport and analysis, a fresh silicon wafer was used for every bi-layer or mixedfilm experiment.

\section{RESULTS}

\section{A. Co-deposition of molybdenum and silicon}

Two electron beam evaporator sources have been operated simultaneously to co-deposit silicon and molybdenum at different ratios at ambient growth temperature. Approximately, $10 \mathrm{~nm}$ thick films with different molybdenum contents $\left(\mathrm{Mo}_{\mathrm{x}} \mathrm{Si}\right)$ have been produced. With growth near room temperature, the silicide formed is assumed to be governed by the arriving species and less by bulk diffusion events. Only limited atomic mobility on the surface needs to be assumed for the arriving species to form the energetically most favored compound. Three stable $\mathrm{Mo}_{\mathrm{X}} \mathrm{Si}$ compounds can be found in the literature: ${ }^{23} \mathrm{MoSi}_{2}(\mathrm{x}=0.5), \mathrm{Mo}_{5} \mathrm{Si}_{3}$ $(\mathrm{x}=1.67)$, and $\mathrm{Mo}_{3} \mathrm{Si}(\mathrm{x}=3)$. With growth temperature low compared to the melting temperatures of molybdenum (10\%) and silicon (18\%) and assuming the thermodynamically most favored compound to be formed from the arriving atom flux, three regions can be distinguished. For x smaller than 1 , the $\mathrm{MoSi}_{2}$ compound is favored. For $\mathrm{x}$ between 1 and 2.7, the $\mathrm{Mo}_{5} \mathrm{Si}_{3}$ compound is favored. Finally, for $\mathrm{x}$ larger than 2.7, the $\mathrm{Mo}_{3} \mathrm{Si}$ compound is the favored compound to be formed. This will require some atomic surface mobility, which will be present at room temperature. The small leftovers are assumed to remain in their elemental state.

Figure 1(a) shows the XPS data of the Mo3d peaks of a reference layer of $7 \mathrm{~nm}$ thick polycrystalline molybdenum and of three $\mathrm{Mo}_{x} \mathrm{Si}$ films with different ratios. The molybdenum peak shifts towards a lower binding energy for the films with a lower metal content. Figure 1(b) depicts the Si2p peaks of a reference layer of $10 \mathrm{~nm}$ thick amorphous silicon and the $\mathrm{Mo}_{\mathrm{x}} \mathrm{Si}$ films corresponding to Figure 1(a). There is no clear trend in the silicon peak position with molybdenum content, although small shifts are observed. In order to assess the binding energy more accurately, both the Mo3d and Si2p peaks are fitted with their respective spin-orbit states. Where for molybdenum the states are clearly separated, for silicon, they are only visible in the asymmetric shape of the Si2p peak. In both cases, the textbook values for the positional difference and peak intensity ratios are used. ${ }^{24}$ Furthermore, the Mo3d $\mathrm{d}_{5 / 2}$ peak shows an asymmetry typical for metal peaks under XPS observation. This asymmetry is located at binding energies higher than the elemental peak position and is taken into account in determining the asymmetry for the reference layer and compensating with the background level. This asymmetric shape is assumed to be present for all molybdenum peaks and only has a minor impact on the peak position or on the peak area. The peak position associated with molybdenum in the elemental state $\left(\mathrm{Mo}_{5 / 2}\right)$ is $227.9 \mathrm{eV}$ and that of silicon in the elemental state $\left(\mathrm{Si} 2 \mathrm{p}_{3 / 2}\right)$ is $99.1 \mathrm{eV}$. Both are obtained from the reference layers.

In order to establish possible chemical shifts in the peak positions of silicon and molybdenum, the binding energy difference with respect to the elemental peak position is plotted in Figure 2. On the horizontal axis, the metal ratio is plotted based on the XPS measurement. This ratio is consistent with the deposited amount of molybdenum and silicon monitored during production of these mixed films. Furthermore, a $1 \mu \mathrm{m}$ thick film of $\mathrm{MoSi}_{2}$ reference material has been characterized and is plotted in Figure 2 as well (open symbol). The reference material has been exposed to the atmosphere, and, consequently, the $\mathrm{MoSi}_{2}$ compound peaks needed to be decomposed from their oxides, decreasing the accuracy of the peak position. This reaction with the atmosphere is the main reason for conducting this study of compound

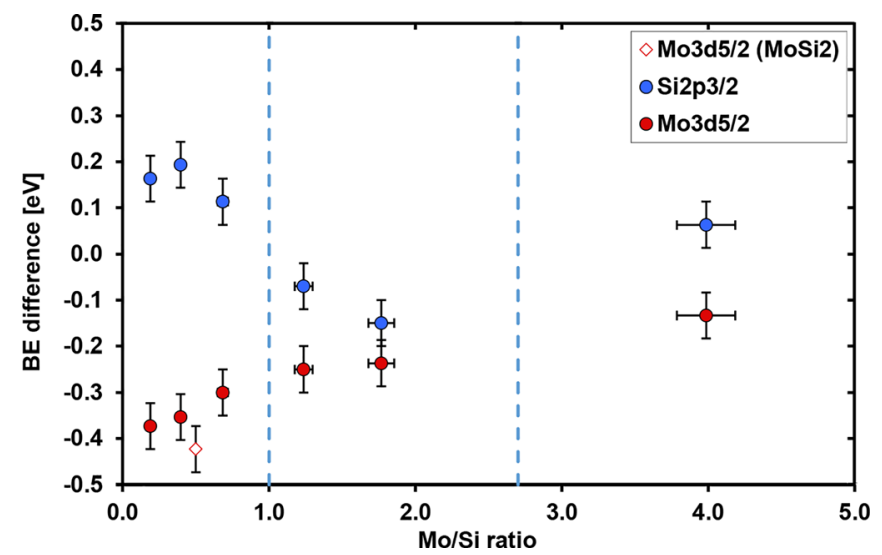

FIG. 2. Chemical shift of silicon and molybdenum binding energy for different $\mathrm{Mo} / \mathrm{Si}$ ratios in mixed layers. 
formation underneath the nanometer thin layers in a vacuum. In Figure 2, the Mo3d $\mathrm{d}_{5 / 2}$ position shows the largest shift for the lowest metal concentration, $-0.4 \mathrm{eV}$, overlapping with the $\mathrm{MoSi}_{2}$ reference at $\mathrm{x}=0.5$. This value is consistent with literature references on $\mathrm{MoSi}_{2}{ }^{13,25}$ The $\mathrm{Si} 2 \mathrm{p}_{3 / 2}$ position corresponding to the $\mathrm{MoSi}_{2}$ compound (around $\mathrm{x}=0.5$ ) shows a chemical shift of $+0.15 \mathrm{eV}$ with respect to the elemental position. A small positive chemical shift can be found in the literature as well. ${ }^{25}$ In Figure 2, the center region, corresponding to a $\mathrm{Mo}_{5} \mathrm{Si}_{3}$ compound, shows a slightly different chemical shift. Here, the Mo3d $\mathrm{d}_{5 / 2}$ has shifted $-0.25 \mathrm{eV}$, and the $\mathrm{Si} 2 \mathrm{p}_{3 / 2}$ shows a $-0.15 \mathrm{eV}$ shift. Even at an $\mathrm{x}$ value larger than 3 , the region corresponding to $\mathrm{Mo}_{3} \mathrm{Si}$, the $\mathrm{Mo}_{3 / 2}$ position is shifted $0.13 \mathrm{eV}$ towards lower binding energy. The silicon peak shift is shown here to be almost absent, but the low intensity makes an accurate estimate difficult.

These shifts are used in the rest of the paper to identify the presence of $\mathrm{Mo}_{x} \mathrm{Si}$ at the interface of the bi-layer systems. Before this can be done, the peak shapes, most importantly the peak width, need to be discussed. The width of an XPS peak is determined by many factors of which the used source and detector are the main ingredients. All reported data in this paper have been recorded with the same source and detector settings. Furthermore, peaks are broadened due to the amorphous nature of some materials compared to their crystalline state. ${ }^{26}$ In this work, the full-width-half-maximum (fwhm) values of amorphous and crystalline silicon for the $\mathrm{Si} 2 \mathrm{p}_{3 / 2}$ peak are $0.8 \mathrm{eV}$ and $0.6 \mathrm{eV}$, respectively, showing the latitude of the structure on the peak width. From the codeposition results, a similar broadening is observed for molybdenum when compared to the large grain (typical grain dimensions $10 \mathrm{~nm}$ ) polycrystalline reference film. While for the reference film, the Mo3d $\mathrm{d}_{5 / 2}$ shows a fwhm of $0.6 \mathrm{eV}$, the co-deposited results show a width of $0.7-0.8 \mathrm{eV}$. With the ambient growth temperature used here, it is very well possible that the evolving $\mathrm{MoSi}_{\mathrm{x}}$ compound occupies a structure with grains small enough to be considered amorphous. In Sections III B and IIIC, the full width of a peak becomes important when small amounts of compound need to be identified together with their bulk species. The amount of a compound is identified by the chemical shift of the Mo3d $\mathrm{d}_{5 / 2}$ peak only, because the molybdenum peak's photo-ionization probability is an order of magnitude higher than for silicon. Furthermore, the fwhm of a possible compound is assumed the same for all compounds and is fixed at a width of $0.7 \mathrm{eV}$, which is the smallest value observed in the mixed films.

\section{B. Bi-layer systems: Mo-on-Si}

Amorphous silicon substrate layers are prepared in a vacuum before covering it with different amounts of molybdenum. The deposited molybdenum thickness $(t)$ is plotted in Figure 3 together with the $\mathrm{Mo}_{3} \mathrm{~d}_{5 / 2}$ chemical shift. In order to validate the reported $\pm 0.05 \mathrm{eV}$ accuracy of the chemical shift, the peak position of $\mathrm{Si}_{2} \mathrm{p}_{3 / 2}$ of the silicon substrate layer is used as a reference. The deposited amount is determined during preparation using a quartz micro mass balance at bulk density $(10.2 \mathrm{~g} / \mathrm{cc}$ for molybdenum). All molybdenum amounts below $\mathrm{t}=2.5 \mathrm{~nm}$ exhibit a chemical shift, suggesting

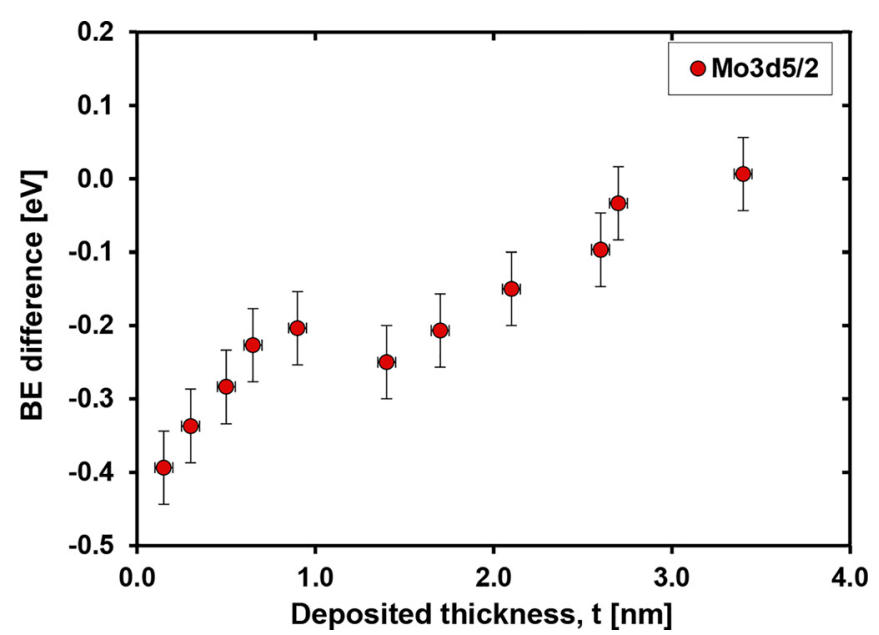

FIG. 3. Chemical shift of molybdenum for different deposited thicknesses of molybdenum on a silicon substrate layer.

a compound state for all molybdenum. Starting at a shift of $-0.4 \mathrm{eV}$ for $\mathrm{t}=0.2 \mathrm{~nm}$, it decreases to a shift of $-0.25 \mathrm{eV}$ around $\mathrm{t}=0.7 \mathrm{~nm}$. Between $\mathrm{t}=0.7 \mathrm{~nm}$ and $\mathrm{t}=2 \mathrm{~nm}$, the shift remains constant around $-0.25 \mathrm{eV}$. The chemical shifts presented in Figure 3 are similar to the results reported by Slaughter et al. ${ }^{13}$ on crystalline silicon, suggesting that the amorphous nature of the silicon substrate layer used here is irrelevant for the range $\mathrm{t}=0-2 \mathrm{~nm}$ covered in both the studies.

Before continuing with a more detailed in-depth modelling of the ARXPS results, the morphology of the films needs to be considered. The surface morphology can change the angular response significantly, ${ }^{20,27-30}$ depending on both the lateral dimensions and the magnitude of the height fluctuations. This study has been carried out on super-polished silicon wafers that are relatively flat. Nevertheless, height fluctuations on the nanometer scale can be expected. The sensitivity of the angular response of XPS to the morphology is determined by the angular binning of the detector. In this paper, the same division of analyzer angles $\left(7.5^{\circ}\right.$ bins of the $60^{\circ}$ acceptance angle) is used as reported previously on angular photo-electron spectroscopy measurements of nanometer thin overlayers. ${ }^{20}$ In this case, the surface morphology similar to that of the super-polished wafer is smooth enough to be modelled with a top layer without taking roughness into account. Figure 4 shows the morphologies of the silicon substrate layer and that of about $3 \mathrm{~nm}$ deposited molybdenum on top of this substrate layer. The in vacuum characterization ensures that the system under observation of the STM is the same as observed by ARXPS. The surface morphology of the substrate layer is quite similar to that of the bi-layer. In both cases, height differences show an isotropic landscape, with a root-mean-square of $0.20 \mathrm{~nm}$ for the silicon layer and $0.23 \mathrm{~nm}$ for the molybdenum layer. These values are similar to the reported value for the super-polished wafer ${ }^{20}$ and can, therefore, be considered smooth enough for XPS modelling without roughness influencing the result. The evolution of the morphology of this system is described elsewhere ${ }^{12}$ and is not discussed in this paper.

To identify the composition of the molybdenum-silicon compound, de-convoluting XPS peaks are required. For a 

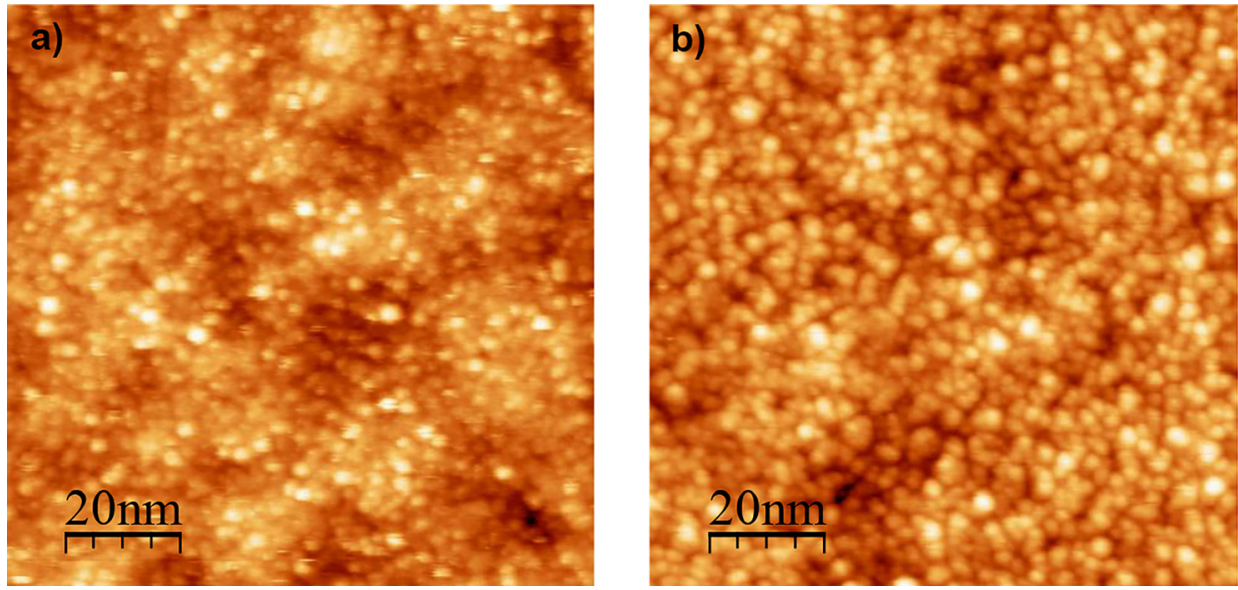

FIG. 4. $100 \mathrm{~nm} \times 100 \mathrm{~nm}$ STM image of (a) initial silicon substrate layer surface $(\mathrm{rms} 0.20 \mathrm{~nm})$ and (b) $3 \mathrm{~nm}$ molybdenum deposited on this initial silicon surface ( $\mathrm{rms} 0.23 \mathrm{~nm})$. reliable composition, both the peak position and the peak width of a chemical state need to be known. This is especially true when the compound is a minority fraction of the total, as is depicted in Figure 5 where the components of the Mo3d peak for the system $\mathrm{Si} / \mathrm{Mo}(2 \mathrm{~nm})$ are displayed. Whereas the $\mathrm{MoSi}_{2}$ component has a fixed chemical shift of $-0.4 \mathrm{eV}$ and a fixed width of $0.7 \mathrm{eV}$, the peak for the Mo3d(top) is allowed an optimum position to fit best. The resulting envelope is shown together with the measurement next to the components and shows a good fit. Also plotted is a small peak labelled $\mathrm{Mo} 3 \mathrm{~d}(\mathrm{O} 2)$ that occupies the position of the $\mathrm{MoO}_{2}$ compound. Where a small amount of metallic oxygen (in the O1s peak) is detected in the XPS measurements corresponding to roughly a monolayer coverage, the area of the $\mathrm{Mo} 3 \mathrm{~d}(\mathrm{O} 2)$ peak is twice the amount metallic oxygen would allow for a $\mathrm{MoO}_{2}$ compound. The $\operatorname{Mo} 3 \mathrm{~d}(\mathrm{O} 2)$ peak is, therefore, partly attributed to the background signal. This peak is omitted from quantification since the compound of interest, $\mathrm{Mo}_{\mathrm{x}} \mathrm{Si}$, is identified at the lower binding energy positions where background signal is unambiguous. Furthermore, a monolayer of metallic oxide can be expected on a molybdenum layer with the used vacuum transport time $(5 \mathrm{~min})$ and base pressure $\left(1 \times 10^{-9}\right.$ mbar).

The model used for quantifying the ARXPS results is a simple three layer model and described in detail in the supplementary material. ${ }^{22}$ From the vacuum interface downwards, the model consists of a top molybdenum layer, a $\mathrm{MoSi}_{2}$ interface layer, and a silicon substrate layer. In order to assign a layer thickness to XPS data modelling, a material density needs to be assumed. For molybdenum and silicon, bulk densities are applied, and for the compound $\mathrm{MoSi}_{2}$, a density of $6.24 \mathrm{~g} / \mathrm{cm}^{3}$ is used. Detector angles up to $60^{\circ}$ are used for which the good fit quality of the used model to the measured angular response confirms the in-depth order of the layers. Figure 6 shows the thickness results obtained from best fit of the box-model to the data with only the compound fraction of the deposited material as the parameter (see supplementary material for details). The reported total thickness is the sum of the molybdenum top layer and the compound interface layer. The model total thickness is inherently consistent with the monitored amount of the material during preparation and is presented to illustrate the timing of the top layer evolution. The evolution of the $\mathrm{MoSi}_{2}$ interface thickness shows a more complex behavior. A MoSi 2 interface thickness of $0.25 \mathrm{~nm}$ evolves instantly when $0.1 \mathrm{~nm}$ molybdenum is deposited. This interface thickness remains intact up to about $\mathrm{t}=1.0 \mathrm{~nm}$. This amount of interface has incorporated 1 monolayer (accuracy of $+/-10 \%$ ) of the substrate silicon, suggesting that mainly the former vacuum interface of the silicon substrate layer has reacted. In the range $\mathrm{t}=1.0-2.0 \mathrm{~nm}$, the $\mathrm{MoSi}_{2}$ thickness is increased with respect to the initial amount to a $1.8 \mathrm{~nm}$ thick interface layer. This amount of interface now incorporates
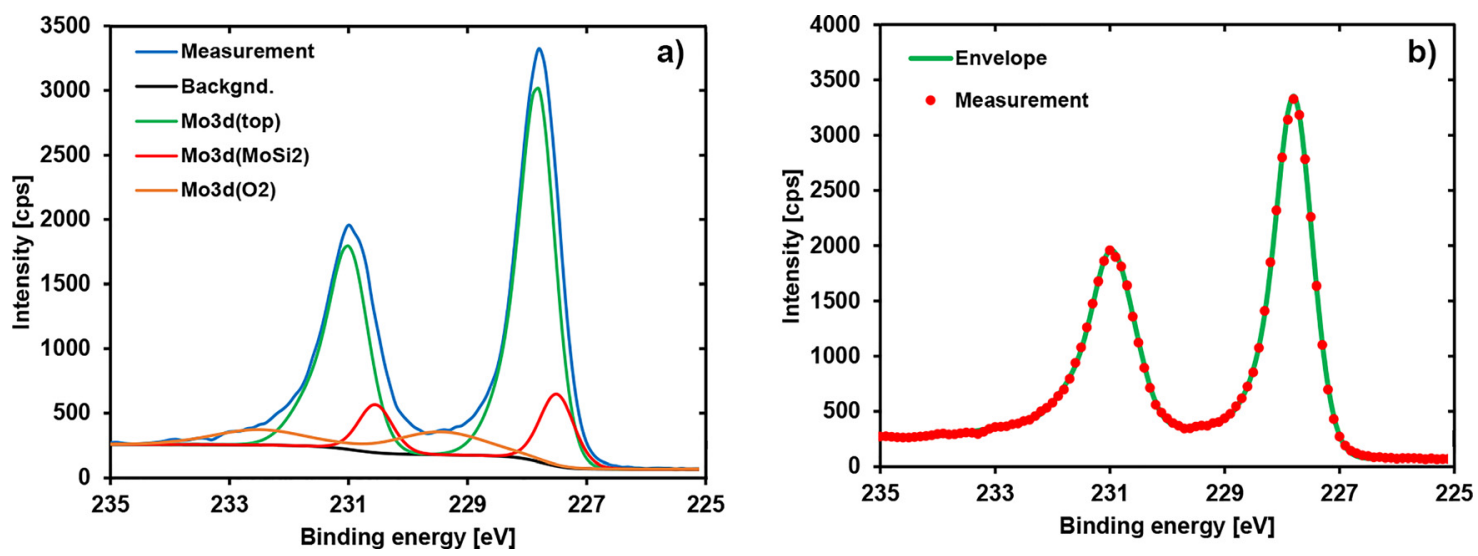

FIG. 5. Si/Mo(2 nm) decomposition of the Mo3d peak in (a) the individual components and in (b) the match between the measured data points and the envelope of the fit. 


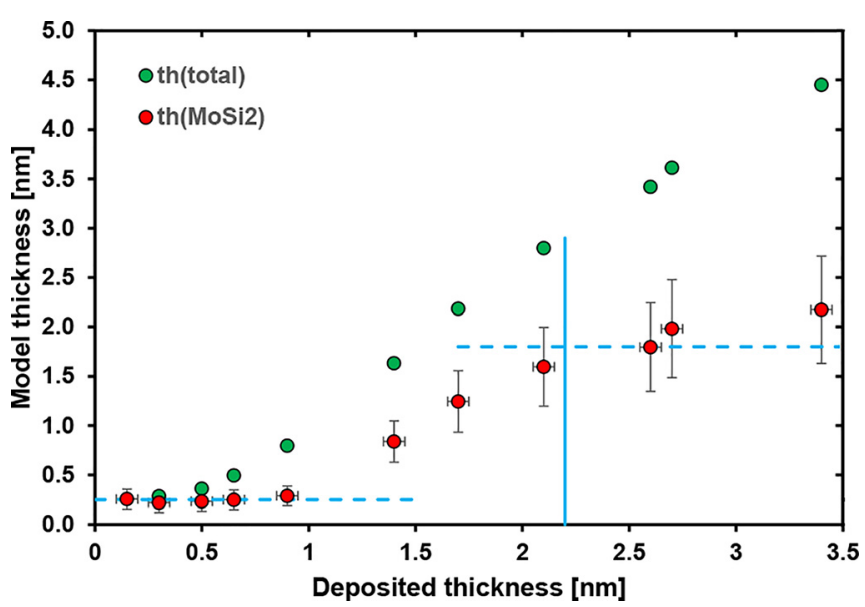

FIG. 6. Thicknesses obtained from the three layer modelling of the ARXPS data. The total thickness matches the total deposited amount, whereas the evolution of the $\mathrm{MoSi}_{2}$ interface thickness between the molybdenum and silicon shows a non-linear behavior: the initial $0.25 \mathrm{~nm}$ interface thickness evolves into a $1.8 \mathrm{~nm}$ interface thickness. The solid blue line indicates the approximate deposited thickness where the $\mathrm{MoSi}_{2}$ interface formation saturates.

around 7 monolayers of substrate silicon, suggesting significant in-depth material redistribution. In this range, the $\mathrm{MoSi}_{2}$ interface thickness accounts for most of the added film thickness. For amounts larger than $\mathrm{t}=2.0 \mathrm{~nm}$, the $\mathrm{MoSi}_{2}$ interface thickness is no longer increasing (indicated by the blue solid line). Besides the thickness dependent behavior of the $\mathrm{MoSi}_{2}$ at the interface, the chemical shift of the top molybdenum layer also holds information. For deposited amounts below $2.0 \mathrm{~nm}$, the top layer is in a compound state as well. A shift of $-0.20 \mathrm{eV}$ suggests this top layer to be $\mathrm{Mo}_{5} \mathrm{Si}_{3}$, although a mixed $\mathrm{Mo}_{5} \mathrm{Si}_{3}-\mathrm{Mo}_{3} \mathrm{Si}$ composition cannot be excluded. Only after $\mathrm{t}=2.5 \mathrm{~nm}$, the top molybdenum layer exhibits a peak signature of bulk poly-crystalline molybdenum. The combined results suggest a complex molybdenum silicide to exist below $\mathrm{t}=2.5 \mathrm{~nm}$.

According to the in-depth analysis of ARXPS data, the evolution of this molybdenum compound layer is a stepwise process. The first sub-monolayer amount of molybdenum reacts with the former vacuum interface of the silicon substrate layer to form a $\mathrm{MoSi}_{2}$ compound. This amount remains constant up to $1.0 \mathrm{~nm}$ deposited molybdenum (4 monolayers). After the initial $0.25 \mathrm{~nm}$ thick $\mathrm{MoSi}_{2}$ compound formation, the arriving molybdenum contributes to the formation of a (most likely) $\mathrm{Mo}_{5} \mathrm{Si}_{3}$ top layer. Between $1.0 \mathrm{~nm}$ and $2.0 \mathrm{~nm}$ deposited molybdenum, the arriving molybdenum contributes mainly to an increase of the $\mathrm{MoSi}_{2}$ compound at the silicon substrate layer's interface, keeping the amount of $\mathrm{Mo}_{5} \mathrm{Si}_{3}$ compound in this range almost constant. Only at larger amounts of 2.5 to $3 \mathrm{~nm}$ deposited, a more straightforward model of bulk molybdenum on the top of a $1.8 \mathrm{~nm}$ thick $\mathrm{MoSi}_{2}$ interface layer emerges. The results show that the interface between molybdenum and silicon evolves underneath the top layer at growth temperatures as low as room temperature. To confirm that the interface formation is mainly deposited amount dependent and not time dependent, a bi-layer at $\mathrm{t}=2.5 \mathrm{~nm}$ has been produced with a factor 10 slower deposition rate of the molybdenum. The
$\mathrm{MoSi}_{2}$ interface thickness of this slower bi-layer matches within the $10 \%$ accuracy typical for these measurements. The exact pathways through which the compounds are formed are beyond the scope of this paper, but first results on the atomic details of this system have already been provided by Fokkema. ${ }^{31}$ In his thesis on a STM study of molybdenum on crystalline silicon, it is clearly shown that initial molybdenum deposition modifies the underlying silicon surface and opens up the silicon facets, providing new pathways for materials diffusion.

\section{Bi-layer systems: Si-on-Mo}

In order to investigate the reverse interface, molybdenum substrate layers are prepared in a vacuum before covering them with different amounts of silicon. In order to avoid the signal intensities of the supporting silicon wafer, $7 \mathrm{~nm}$ thick (poly-)crystalline layers are used as a substrate layer. To validate the reported $\pm 0.05 \mathrm{eV}$ accuracy of the chemical shift, the peak position of Mo3d of the molybdenum substrate layer is used as a reference. The $\mathrm{Si} 2 \mathrm{p}_{3 / 2}$ chemical shift for different silicon top layers up to $6 \mathrm{~nm}$ thickness is not showing any significant shift with respect to a silicon reference film. Only for the smallest deposited amount of $0.15 \mathrm{~nm}$, the chemical shift in the silicon peak is significantly different from reference amorphous silicon. The shift of $-0.2 \mathrm{eV}$ would suggest a $\mathrm{Mo}_{5} \mathrm{Si}_{3}$ compound based on the compound identification proposed in section "co-deposition." In order to have a closer look at compound formation in the system silicon on molybdenum, the same approach as described in Section III B is deployed.

Before using ARXPS modelling, the surface morphologies of these bi-layers are probed. Figure 7 shows the results of the molybdenum substrate layer and $6 \mathrm{~nm}$ deposited silicon on top of this substrate. The surface morphology of the substrate layer is similar to that of the bi-layer. In both the cases, the height differences show an isotropic landscape with a root-mean-square of $0.44 \mathrm{~nm}$ for the molybdenum layer and $0.51 \mathrm{~nm}$ for the silicon layer. These values are larger than the reported value for the super-polished wafer ${ }^{20}$ and can, therefore, no longer be considered flat for XPS modelling. The picture also shows that lateral correlation lengths (from height-difference correlation) are slightly different with $2.3 \mathrm{~nm}$ and $3.5 \mathrm{~nm}$ for the molybdenum and silicon layers, respectively. These are of the same order of magnitude as the attenuation lengths of XPS. Consequently, modelling ARXPS data will be influenced similar for both the morphologies. The extent of height differences in these systems prevents the unique identification of the in-depth layer position from ARXPS data ${ }^{20}$ for this bi-layer, but a simple three layer model can still be used. Provided the assumed in-depth layer order is right and omitting the most grazing detector angles, in this case, all angles above $45^{\circ}$, a compound interface amount can be estimated when the compound can be identified via a chemical shift. In the system silicon-on-molybdenum, the Mo3 $\mathrm{d}_{5 / 2}$ peak is again used to identify the possible compound formation. With the peak shape (mainly position and fwhm) of the substrate layer known, a peak broadening of the envelope can be assigned 

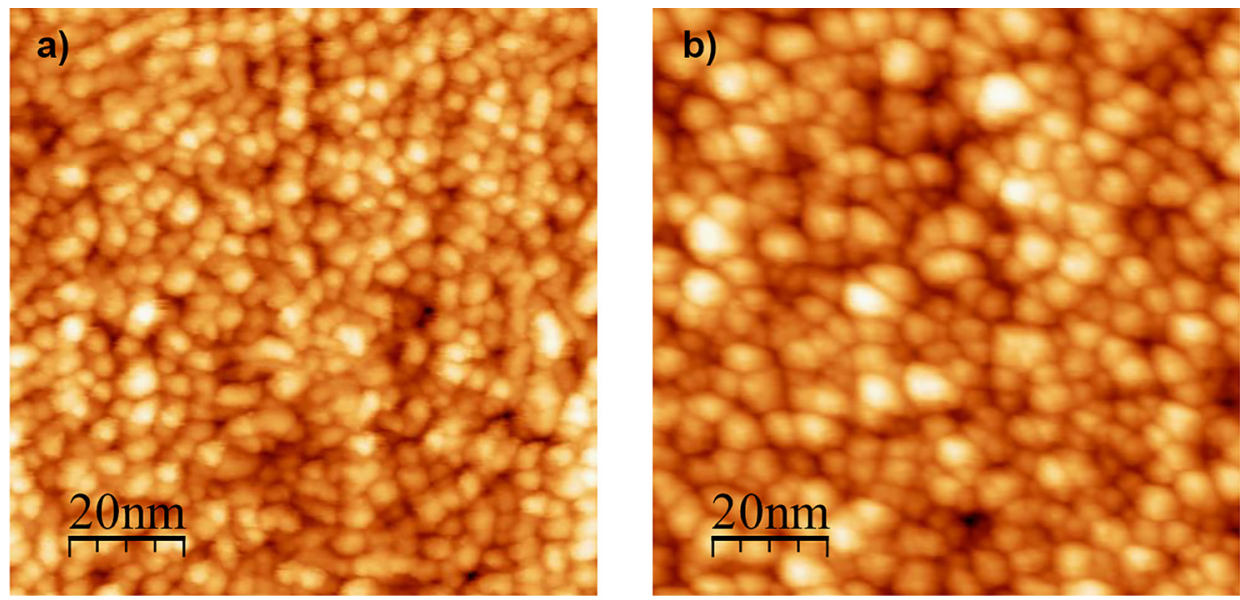

FIG. 7. $100 \mathrm{~nm} \times 100 \mathrm{~nm}$ STM pictures of (a) the molybdenum substrate layer $(\mathrm{rms} 0.44 \mathrm{~nm})$ and (b) $6 \mathrm{~nm}$ silicon deposited on the molybdenum substrate layer $(\mathrm{rms} 0.51 \mathrm{~nm})$. to the compound formation. The molybdenum peak composition is assumed to consist of three components, a bulk molybdenum component with bulk peak constrains, a $\mathrm{MoSi}_{2}$ component with a chemical shift of $-0.4 \mathrm{eV}$ and a fwhm of $0.7 \mathrm{eV}$, and $\mathrm{a} \mathrm{Mo}_{5} \mathrm{Si}_{3}$ component with a chemical shift of $-0.25 \mathrm{eV}$ and a fwhm of $0.7 \mathrm{eV}$. For all the deposited silicon amounts, the best fit of the molybdenum peak shows the $\mathrm{MoSi}_{2}$ component to be only a marginal fraction (smaller than $0.1 \mathrm{~nm}$ ). Therefore, this component is omitted in the quantification. From the vacuum interface down, the model consists of a top silicon layer, a $\mathrm{Mo}_{5} \mathrm{Si}_{3}$ interface layer, and a molybdenum substrate layer. To assign a layer thickness to the $\mathrm{Mo}_{5} \mathrm{Si}_{3}$ compound, a density of $8.24 \mathrm{~g} / \mathrm{cm}^{3}$ is assumed. The resulting layer thicknesses are plotted in Figure 8. The amount of interface layer thickness is significantly smaller for this bi-layer compared to that described in Section III B. Below $\mathrm{t}=0.8 \mathrm{~nm}$, the $\mathrm{Mo}_{5} \mathrm{Si}_{3}$ interface is only $0.25 \mathrm{~nm}$ thick. For deposited amounts above $\mathrm{t}=1.3 \mathrm{~nm}$, the interface layer thickness saturates at $0.4 \mathrm{~nm}$. This is roughly a quarter of the thickness of the molybdenum-on-silicon of Section III B. The initial $\mathrm{Mo}_{5} \mathrm{Si}_{3}$ interface thickness of $0.25 \mathrm{~nm}$ has consumed only 0.7 monolayers of the molybdenum substrate. Consequently, the final interface layer thickness consumes

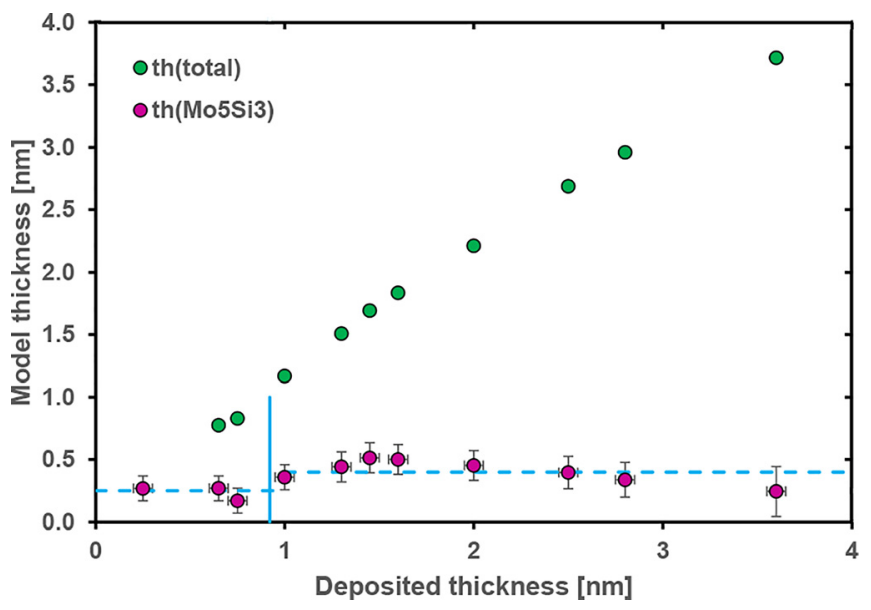

FIG. 8. Thicknesses obtained from the three layer modelling of the ARXPS data. The silicon thickness matches the total deposited amount, whereas the $\mathrm{Mo}_{5} \mathrm{Si}_{3}$ interface evolves underneath the silicon top layer. The solid blue line indicates the approximate deposited thickness where the $\mathrm{Mo}_{5} \mathrm{Si}_{3}$ interface formation saturates.
1.0 monolayer worth of substrate atoms. Although on a smaller scale, this buried interface shows evolution underneath the nanometer thick layers as well.

\section{SUMMARY AND DISCUSSION}

All facets of angle resolved x-ray photoelectron spectroscopy (ARXPS) have been employed to determine nondestructively the molybdenum-silicon compound formation at buried interfaces. Thin compound films are produced and analyzed in vacuo to identify the chemical shifts of the $\mathrm{Mo}_{5 / 2}$ and $\mathrm{Si} 2 \mathrm{p}_{3 / 2}$ peaks. The $\mathrm{MoSi}_{2}, \mathrm{Mo}_{5} \mathrm{Si}_{3}$, and $\mathrm{Mo}_{3} \mathrm{Si}$ compounds can be distinguished from their elemental state by careful analysis of the molybdenum peak. Buried interface amounts are estimated by using the chemical shift identification of the compounds and quantifying ARXPS results. Room temperature deposition of molybdenum and silicon bilayers shows an evolution of the interface depending on the deposited amount of top layer material. This indicates both the meta-stable state of the initially formed interface as well as the potential for (limited) in-depth materials transport across the interface.

The Mo-on-Si interface shows two distinct in-depth compositions, depending on the deposited amount. For the molybdenum layer thickness above $2.5 \mathrm{~nm}$, a (poly-)crystalline top layer exists on top of a $1.8 \mathrm{~nm}$ thick $\mathrm{MoSi}_{2}$ interface layer. For smaller amounts, all molybdenum is in a compound state. This compound state consists of a $0.25 \mathrm{~nm}$ thick $\mathrm{MoSi}_{2}$ part at the interface, with the silicon substrate layer and a molybdenum rich top layer. Around $2.0 \mathrm{~nm}$ deposited (8 monolayers), the molybdenum compound layer consists of about $1.8 \mathrm{~nm} \mathrm{MoSi} i_{2}$ and $1 \mathrm{~nm} \mathrm{Mo} \mathrm{Mi}_{5}$. With increasing molybdenum amounts, the $\mathrm{MoSi}_{2}$ remains at $1.8 \mathrm{~nm}$ layer thickness, while the molybdenum rich top layer transforms to (poly-)crystalline molybdenum after $2.5 \mathrm{~nm}$ deposited (10 monolayers). The saturation of $\mathrm{MoSi}_{2}$ formation seems prerequisite before the top layer can crystalize. ${ }^{12}$ This deposition amount dependent complex behavior of interface formation at room temperature requires a significant interaction of the silicon substrate layer and the molybdenum top layer. This can be achieved by diffusion of atomic species across the interface together with morphology changes due to compound formation. 
The Si-on-Mo interface shows a smaller amount of interface formation than the Mo-on-Si interface, but a deposited amount dependent evolution is observed nevertheless. An initial $\mathrm{Mo}_{5} \mathrm{Si}_{3}$ interface of $0.25 \mathrm{~nm}$ thickness is formed from the arriving silicon. This amount consumes about 0.7 monolayers of the molybdenum substrate layer. Only when the silicon deposition exceeds $1.0 \mathrm{~nm}$, the interface thickness increases to $0.4 \mathrm{~nm}$. This interface thickness remains constant with increasing amount.

Comparing compound formation at both the interfaces, the Mo-on-Si interface has a larger impact on the substrate layer, consuming up to 7 monolayers worth of a substrate material. This is significantly more than at the Si-on-Mo interface where 1.0 monolayer of the substrate material is consumed. The relatively larger amount of the substrate material transformed for the Mo-on-Si interface compared to the reverse interface can partly be explained by the amorphous nature of the silicon substrate layer. This allows easier binding sites for the arriving atoms due to more dangling bonds when compared to the (poly-)crystalline texture of the molybdenum substrate layer of the reverse interface. This argument is especially true for the first arriving atoms. The main promotor for $\mathrm{MoSi}_{2}$ compound formation on the Moon-Si interface is, however, found when deposition exceeds $1.0 \mathrm{~nm}$. Where the reverse interface shows hardly any increase in the compound amount over the initial amount, the Mo-on-Si interface continues its transformation towards more $\mathrm{MoSi}_{2}$ compounds. This can only happen when (part of) the compound formation energy is used to free up a fresh, unreacted substrate material. This could occur by, for example, creating small clusters of $\mathrm{MoSi}_{2}$ that partly cover the substrate layer. The saturation point would now indicate the stage where these clusters of $\mathrm{MoSi}_{2}$ cover the substrate layer sufficiently to block further transport of materials for compound formation. This process is clearly absent on the reverse Si-on-Mo interface.

For EUV multilayers, this mixed composition of the interface region will reduce its performance. The amount of molybdenum and silicon in the multilayers in every period is about $3 \mathrm{~nm}$ and $5 \mathrm{~nm}$, respectively. At these amounts, the Mo-on-Si interface will consist of $1.8 \mathrm{~nm} \mathrm{MoSi}_{2}$, and the Sion-Mo interface will consist of $0.4 \mathrm{~nm} \mathrm{Mo}{ }_{5} \mathrm{Si}_{3}$. This study is in line with the previously reported values for these multilayers. ${ }^{2,8}$ IMD (software for modeling optical properties of multilayer films) reflectance simulations show that this amount of interface will reduce the maximum EUV reflectance of $75 \%$ with about $5 \%$. The compound formation, therefore, accounts for the majority of the reflectance loss in the observed $69 \%$ surface morphology during the deposition process. ${ }^{12}$

\section{CONCLUSIONS}

Where the presented results show the dynamic interface behavior at relatively low growth temperature (room temperature) in $\mathrm{Mo} / \mathrm{Si}$ multilayer systems, this is but an example for the potential the employed technique harbors for characterizing buried interfaces underneath the nanometer thick layers. Thanks to an in-depth probing volume that is sensitive to both the top as well as the substrate layers and the possibility to identify the compound states via the chemical shifts, angle resolved x-ray photoelectron spectroscopy (ARXPS) is a good tool to start the study of any interface problem at the nanometer scale.

\section{SUPPLEMENTARY MATERIAL}

See supplementary material for the three-layer-model or box-model ${ }^{22}$ used in this paper to quantify the measured angle resolved photoelectron data. It also describes the relation to the reported error bars in more detail.

\section{ACKNOWLEDGMENTS}

Part of this work was carried out in the framework of the STW Program "Nano-engineering rules for X-ray and EUV optics: Atomic-scale controlled deposition," which was carried out in cooperation with Leiden University. Furthermore, this work was part of the FOM Industrial Partnership Program Nos. I10 ("XMO") and I23 (“CP3E"), which were carried out under contract with Carl Zeiss SMT $\mathrm{GmbH}$, Oberkochen, ASML, Veldhoven, and the "Stichting voor Fundamenteel Onderzoek der Materie (FOM)," the latter being financially supported by the "Nederlandse Organisatie voor Wetenschappelijk Onderzoek (NWO)."

${ }^{1}$ E. Louis, H. J. Voorma, N. B. Koster, L. Shmaenok, F. Bijkerk, R. Schlatmann, J. Verhoeven, Y. Y. Platonov, G. E. van Dorssen, and H. A. Padmore, Microelectron. Eng. 23(1-4), 215-218 (1994).

${ }^{2}$ I. Nedelcu, R. W. E. van de Kruijs, A. E. Yakshin, F. Tichelaar, E. Zoethout, E. Louis, H. Enkisch, S. Muellender, and F. Bijkerk, Thin Solid Films 515(2), 434-438 (2006).

${ }^{3}$ R. Schlatmann, C. Lu, J. Verhoeven, E. J. Puik, and M. J. van der Wiel, Appl. Surf. Sci. 78(2), 147-157 (1994).

${ }^{4}$ E. Louis, E. D. V. Hattum, S. A. V. D. Westen, P. Salle, K. T. Grootkarzijn, E. Zoethout, F. Bijkerk, G. V. Blanckenhagen, and S. Mullender, Proc. SPIE 7636, 76362 T (2010).

${ }^{5}$ E. Louis, A. E. Yakshin, T. Tsarfati, and F. Bijkerk, Prog. Surf. Sci. 86(11-12), 255-294 (2011).

${ }^{6}$ K. Le Guen, H. Maury, J. M. André, H. Wang, J. Zhu, Z. Wang, and P. Jonnard, Appl. Surf. Sci. 253(20), 8443-8446 (2007).

${ }^{7}$ I. Nedelcu, R. W. E. van de Kruijs, A. E. Yakshin, and F. Bijkerk, Phys. Rev. B 76(24), 245404 (2007).

${ }^{8}$ R. S. Rosen, D. G. Stearns, M. A. Viliardos, M. E. Kassner, S. P. Vernon, and Y. D. Cheng, Appl. Opt. 32(34), 6975-6980 (1993).

${ }^{9}$ H. J. Voorma, E. Louis, F. Bijkerk, and S. Abdali, J. Appl. Phys. 82(4), 1876-1881 (1997).

${ }^{10}$ H. J. Voorma, E. Louis, N. B. Koster, and F. Bijkerk, J. Appl. Phys. 83(9), 4700-4708 (1998).

${ }^{11}$ A. J. R. van den Boogaard, E. Zoethout, I. A. Makhotkin, E. Louis, and F. Bijkerk, J. Appl. Phys. 112(12), 123502 (2012).

${ }^{12}$ E. Zoethout, E. Louis, and F. Bijkerk, Appl. Surf. Sci. 285, 293-299 (2013)

${ }^{13}$ J. M. Slaughter, A. Shapiro, P. A. Kearney, and C. M. Falco, Phys. Rev. B 44(8), 3854-3863 (1991).

${ }^{14}$ P. J. Bedrossian, Surf. Sci. 322(1-3), 73-82 (1995).

${ }^{15}$ H. R. Kaufman, Fundamentals of Ion-Source Operation (Alexandria, Virginia, USA, 1984).

${ }^{16}$ J. H. Scofield, J. Electron Spectrosc. Relat. Phenom. 8(2), 129-137 (1976).

${ }^{17}$ P. J. Cumpson and M. P. Seah, Surf. Interface Anal. 25(6), 430-446 (1997).

${ }^{18}$ J. P. Chang, M. L. Green, V. M. Donnelly, R. L. Opila, J. J. Eng, J. Sapjeta, P. J. Silverman, B. Weir, H. C. Lu, T. Gustafsson, and E. Garfunkel, J. Appl. Phys. 87(9), 4449-4455 (2000).

${ }^{19}$ R. Q. Tan, Y. Azuma, T. Fujimoto, J. W. Fan, and I. Kojima, Surf. Interface Anal. 36(8), 1007-1010 (2004).

${ }^{20}$ E. Zoethout, Surf. Interface Anal. 46(10-11), 1047-1050 (2014). 
${ }^{21}$ P. J. Cumpson, J. Electron Spectrosc. Relat. Phenomena 73(1), 25-52 (1995).

${ }^{22}$ D. Briggs and J. T. Grant, Surface Analysis by Auger and X-ray Photoelectron Spectroscopy (IMPublications and SurfaceSpectra, Chichester, UK, 2003).

${ }^{23}$ R. B. F. R. de Boer, W. C. M. Mattens, A. R. Miedema, and A. K Niessen, Cohesion in Metals (North-Holland, 1988).

${ }^{24}$ W. F. S. J. F. Moulder, P. E. Sobol, and K. D. Bomben, Handbook of $x$ Ray Photoelectron Spectroscopy (Perkin-Elmer Corporation Physical Electronics, Eden Prairie, 1992).

${ }^{25}$ C. D. Wagner, D. E. Passoja, H. F. Hillery, T. G. Kinisky, H. A. Six, W. T. Jansen, and J. A. Taylor, J. Vac. Sci. Amp. Technol. 21(4), 933-944 (1982).
${ }^{26}$ W. F. Egelhoff, Surf. Sci. Rep. 6, 253-415 (1987).

${ }^{27}$ P. L. J. Gunter, O. L. J. Gijzeman, and J. W. Niemantsverdriet, Appl. Surf. Sci. 115(4), 342-346 (1997).

${ }^{28}$ P. Kappen, K. Reihs, C. Seidel, M. Voetz, and H. Fuchs, Surf. Sci. 465(1-2), 40-50 (2000).

${ }^{29}$ K. Olejnik, J. Zemek, and W. S. M. Werner, Surf. Sci. 595(1-3), 212-222 (2005).

${ }^{30}$ W. S. M. Werner, Surf. Interface Anal. 23(10), 696-704 (1995).

${ }^{31}$ V. Fokkema, Ph.D. thesis (Leiden University, Universiteit Leiden, 2011).

${ }^{32}$ D. L. Windt, Comput. Phys. 12(4), 360-370 (1998). 